\title{
Birman-Wenzl-Murakami Algebra, Topological parameter and Berry phase
}

\author{
Chengcheng Zhou, ${ }^{1,} *$ Kang Xue, ${ }^{1,}+{ }_{\dagger}$ Lidan Gou, ${ }^{2,1}$ Chunfang Sun, ${ }^{1}$ Gangcheng Wang, ${ }^{1}$ and Taotao Hu ${ }^{1}$ \\ ${ }^{1}$ School of Physics, Northeast Normal University, Changchun 130024, People's Republic of China \\ ${ }^{2}$ School of Science, Changchun University of Science and Technology, Changchun, 130022, People's Republic of China
}

In this paper, a $3 \times 3$-matrix representation of Birman-Wenzl-Murakami(BWM) algebra has been presented. Based on which, unitary matrices $A\left(\theta, \varphi_{1}, \varphi_{2}\right)$ and $B\left(\theta, \varphi_{1}, \varphi_{2}\right)$ are generated via YangBaxterization approach. A Hamiltonian is constructed from the unitary $B(\theta, \varphi)$ matrix. Then we study Berry phase of the Yang-Baxter system, and obtain the relationship between topological parameter and Berry phase.

PACS numbers: $02.40 .-\mathrm{k}, 03.65 . \mathrm{Vf}$

* Zhoucc237@nenu.edu.cn

† Xuekang@nenu.edu.cn 


\section{INTRODUCTION}

To the best of our knowledge, the Yang-Baxter equation (YBE) was initiated in solving the one-dimensional $\delta$ interecting models [1] and the statistical models [2]. Braid algebra and Temperley-Lieb algebra (TLA) [3] have been widely used in the construction of YBE solutions $[4]$ | and have been introduced to the field of quantum information, quantum computation and topological computation [10 15]. The Birman-Wenzl-Murakami (BWM) algebra [16] which contain two subalgebra (Braid algebra and TLA) was first defined and independently studied by Birman, Wenzl and Murakami. Very recently [17], S.Abramsky demonstrate the connections from knot theory to logic and computation via quantum mechanics. But, the physical meaning of the topological parameter $d$ (describing the single loop in topology) is still unclear.

The geometrical phase [18], such as Berry phase, is an important concept in quantum mechanics [19 24]. In recent years, numerous works have been attributed to Berry phase $|25|$, because of its possible applications to quantum computation (the so-called geometric quantum computation) [26 29]. Quantum logic gates based on geometric phases have been certified in both nuclear magnetic resonance [26] and ion trap based on quantum information architectures [30]. The Ref. [26] pointed out geometric phases have potential fault tolerance when applied to quantum information processing. In 2007, Leek, P.J. et al. [31] illustrated the controlled accumulation of a geometric phase, Berry phase, in a superconducting qubit.

The Ref. [32] applied TLA as a bridge to recast 4-dimensional YBE into its 2-dimensional counterpart. The 2-dimensional YBE have an important application value in topological quantum computation [33, 34]. To date, few studies have reported 3-dimensional YBE which may have potential application values in topological quantum computation. The motivation of this paper is twofold: one is that we structure 3-dimensional YBE, the other is to study the physical meaning of topological parameter $d$ from Berry phase. This paper is organized as follows: In Sec. 2, we introduce a specialized type BWM algebra, and present a $3 \times 3$-matrix representation of BWM algebra. In Sec. 3, we obtain unitary matrices $A\left(\theta, \varphi_{1}, \varphi_{2}\right), B\left(\theta, \varphi_{1}, \varphi_{2}\right)$ via Yang-Baxterization approach. Based on the solution, a Hamiltonian of the Yang-Baxter system is constructed, finally we study the Berry phase of this system. We end with a summary. 


\section{BWM ALGEBRA}

As we know the Braid relations are

$$
\left\{\begin{array}{l}
b_{i} b_{i \pm 1} b_{i}=b_{i \pm 1} b_{i} b_{i \pm 1} \\
b_{i} b_{j}=b_{j} b_{i},|i-j| \geq 2
\end{array}\right.
$$

where $b_{i}=\stackrel{1}{I} \otimes \cdots \otimes \stackrel{i-1}{I} \otimes b \otimes \stackrel{i+2}{I} \otimes \cdots$. When we just consider three tensor product space, the Braid relations becomes

$$
b_{12} b_{23} b_{12}=b_{23} b_{12} b_{23}
$$

where $b_{12}=b \otimes I, b_{23}=I \otimes b, b$-matrix is a $N^{2} \times N^{2}$ matrix acted on the tensor product space $\nu \otimes \nu$, where $N$ is the dimension of $\nu$. It is also well known that the braid relation can be reduced to a $N$-dimensional braid relation $\left(b_{12} \rightarrow A, b_{23} \rightarrow B\right)$

$$
A B A=B A B
$$

Like this reduced method, we easily obtain $N$-dimensional reduced BWM-algebra relations from classical BWMalgebra relations. The BWM algebra [16, 35-37] is generated by the unit $I$, the braid operators $S_{i}$ and the TLA operators $E_{i}$ and depends on two independent parameters $\omega$ and $\sigma$. Let us take the BWM relations as follows.

$$
\left\{\begin{array}{l}
S_{i}-S_{i}^{-1}=\omega\left(I-E_{i}\right) \\
S_{i} S_{i \pm 1} S_{i}=S_{i \pm 1} S_{i} S_{i \pm 1}, S_{i} S_{j}=S_{j} S_{i},|i-j| \geq 2 \\
E_{i} E_{i \pm 1} E_{i}=E_{i}, E_{i} E_{j}=E_{j} E_{i},|i-j| \geq 2 \\
E_{i} S_{i}=S_{i} E_{i}=\sigma E_{i} \\
S_{i \pm 1} S_{i} E_{i \pm 1}=E_{i} S_{i \pm 1} S_{i}=E_{i} E_{i \pm 1} \\
S_{i \pm 1} E_{i} S_{i \pm 1}=S_{i}^{-1} E_{i \pm 1} S_{i}^{-1} \\
E_{i \pm 1} E_{i} S_{i \pm 1}=E_{i \pm 1} S_{i}^{-1}, S_{i \pm 1} E_{i} E_{i \pm 1}=S_{i}^{-1} E_{i \pm 1} \\
E_{i} S_{i \pm 1} E_{i}=\sigma^{-1} E_{i} \\
E_{i}^{2}=\left(1-\frac{\sigma-\sigma^{-1}}{\omega}\right) E_{i}
\end{array}\right.
$$

where $0 \neq d=\left(1-\frac{\sigma-\sigma^{-1}}{\omega}\right) \in \mathbb{C}$ is a topological parameter in knot theory which does not depend on the sites of the lattices. 
By reducing to the $N$-dimensional space $\left(S_{12} \rightarrow A, S_{23} \rightarrow B, E_{12} \rightarrow E_{A}, E_{23} \rightarrow E_{B}\right)$, we have:

$$
\left\{\begin{array}{l}
A-A^{-1}=\omega\left(I-E_{A}\right), B-B^{-1}=\omega\left(I-E_{B}\right) \\
A B A=B A B \\
E_{A} E_{B} E_{A}=E_{A}, E_{B} E_{A} E_{B}=E_{B} \\
E_{A} A=A E_{A}=\sigma E_{A}, E_{B} B=B E_{B}=\sigma E_{B} \\
A B E_{A}=E_{B} A B=E_{B} E_{A}, B A E_{B}=E_{A} B A=E_{A} E_{B} \\
A E_{B} A=B^{-1} E_{A} B^{-1}, B E_{A} B=A^{-1} E_{B} A^{-1} \\
E_{A} E_{B} A=E_{A} B^{-1}, E_{B} E_{A} B=E_{B} A^{-1} \\
A E_{B} E_{A}=B^{-1} E_{A}, B E_{A} E_{B}=A^{-1} E_{B} \\
E_{A} B E_{A}=\sigma^{-1} E_{A}, E_{B} A E_{B}=\sigma^{-1} E_{B} \\
E_{A}^{2}=\left(1-\frac{\sigma-\sigma^{-1}}{\omega}\right) E_{A}, E_{B}^{2}=\left(1-\frac{\sigma-\sigma^{-1}}{\omega}\right) E_{B}
\end{array}\right.
$$

where $A, B$ satisfy the $N$-dimensional Braid relation (3), $E_{A}, E_{B}$ satisfy the $N$-dimensional TLA relations

$$
\left\{\begin{array}{l}
E_{A} E_{B} E_{A}=E_{A}, E_{B} E_{A} E_{B}=E_{B} \\
E_{A}^{2}=d E_{A}, E_{B}^{2}=d E_{B}
\end{array}\right.
$$

It is interesting that Eq.(4) and Eq.(5) have the same topological parameter $d$.

In this paper, the $A$-matrix, $B$-matrix, $E_{a}$-matrix, $E_{b}$-matrix, $A(x)$-matrix and $B(x)$-matrix are $3 \times 3$ matrices acting on the 3-dimensional space. To the TLA relations (6), we assume $E_{A}$ and $E_{B}$ possess the same eigenvalues $d$ and 0 . We assume $E_{A}$ is a diagonal matrix as following

$$
E_{A}=\left(\begin{array}{ccc}
0 & 0 & 0 \\
0 & d & 0 \\
0 & 0 & 0
\end{array}\right)
$$

After tedious calculation,we obtain

$$
E_{B}=\left(\begin{array}{lrr}
\frac{d^{2}-d-1}{d} & \frac{\sqrt{d^{2}-d-1}}{d} e^{i \varphi_{1}} & -\frac{\sqrt{d^{2}-d-1}}{\sqrt{d}} e^{i\left(\varphi_{1}+\varphi_{2}\right)} \\
\frac{\sqrt{d^{2}-d-1}}{d} e^{-i \varphi_{1}} & \frac{1}{d} & -\frac{e^{i \varphi_{2}}}{\sqrt{d}} \\
-\frac{\sqrt{d^{2}-d-1}}{\sqrt{d}} e^{-i\left(\varphi_{1}+\varphi_{2}\right)} & -\frac{e^{-i \varphi_{2}}}{\sqrt{d}} & 1
\end{array}\right)
$$


It is worth to mention that $E_{B}=U E_{A} U^{-1}$, and $U$ is a unitary transformation matrix as follows

$$
U=\left(\begin{array}{lcr}
\frac{1}{(d-1) d} & -\frac{\sqrt{d^{2}-d-1}}{d} e^{i \varphi_{1}} & -\frac{\sqrt{d^{2}-d-1}}{\sqrt{d}(d-1)} e^{i\left(\varphi_{1}+\varphi_{2}\right)} \\
\frac{\sqrt{d^{2}-d-1}}{d} e^{-i \varphi_{1}} & -\frac{1}{d} & \frac{e^{i \varphi_{2}}}{\sqrt{d}} \\
\frac{\sqrt{d^{2}-d-1}}{\sqrt{d}(d-1)} e^{-i\left(\varphi_{1}+\varphi_{2}\right)} & \frac{e^{-i \varphi_{2}}}{\sqrt{d}} & -\frac{d-2}{d-1}
\end{array}\right),
$$

where $d, \varphi_{1}$ and $\varphi_{2}$ are reals. The parameter $d$ is the so-called topological parameter. For simplicity, we just consider the case of $d>0$ in this paper.

The Ref. [35] has explored $S_{i}$ have 3 different eigenvalues $\left(q,-q^{-1}, q^{-2}\right)$ in the BWM-algebra (i.e. Eq.(44)). The same as $E_{A}$ and $E_{B}$, we assume $A$ and $B$ have the same eigenvalues $\left(q,-q^{-1}, q^{-2}\right)$. The simplest $A$ is

$$
A=\left(\begin{array}{rrr}
q & 0 & 0 \\
0 & q^{-2} & 0 \\
0 & 0 & -q^{-1}
\end{array}\right),
$$

using the unitary transformation matrix $U$, we have

$$
B=U A U^{-1}=\left(\begin{array}{lrr}
\frac{1}{q^{4}(d-1) d} & \frac{\sqrt{d^{2}-d-1}}{d q} e^{i \varphi_{1}} & -\frac{\sqrt{d^{2}-d-1}}{q^{2}(d-1) \sqrt{d}} e^{i\left(\varphi_{1}+\varphi_{2}\right)} \\
\frac{\sqrt{d^{2}-d-1}}{d q} e^{-i \varphi_{1}} & \frac{q^{2}}{d} & \frac{q}{\sqrt{d}} e^{i \varphi_{2}} \\
-\frac{\sqrt{d^{2}-d-1}}{q^{2}(d-1) \sqrt{d}} e^{-i\left(\varphi_{1}+\varphi_{2}\right)} & \frac{q}{\sqrt{d}} e^{-i \varphi_{2}} & \frac{d-2}{d-1}
\end{array}\right),
$$

where $d=q^{-1}+1+q$ and the parameter $q$ is real. The matrices $A$ and $B$ satisfy the braid relation $(i . e$. Eq(3) $)$. Towards braid relation, in some models $\varphi_{i},(i=1,2)$, may have a physical significance of magnetic flux. In the paper [13], it has been shown the parameters $\varphi_{i}$ 's are related to Berry phase.

Then we can verify that $\left\{I, A, E_{A}, B, E_{B}\right\}$ satisfy the reduced BWM-algebra (i.e. Eq. (50) $)$, with $d=q^{-1}+1+q$. Here we have set $\omega=q-q^{-1}$ and $\sigma=q^{-2}$. It is interesting that $A, B, E_{A}, E_{B}$ are Hermitian matrices, and have the same similar transformation $B=U A U^{-1}, E_{B}=U E_{A} U^{-1}$, where $U$ is unitary $\left(\right.$ i.e. $\left.U^{\dagger}=U^{-1}\right)$.

\section{YANG-BAXTERIZATION, HAMILTONIAN, BERRY PHASE}

In this section, A Hamiltonian is constructed from the unitary $B(\theta, \varphi)$ matrix. Then we study the Berry phase of the Yang-Baxter system, and obtain the relationship between the topological parameter and the Berry phase. We 
first explain the basic formula of YBE. The Yang-Baxter matrix $\check{R}$ is a $N^{2} \times N^{2}$ matrix acting on the tensor product space $\nu \otimes \nu$, where $N$ is the dimension of $\nu$. Such a matrix $\check{R}$ satisfies the relativistic YBE[32] as follows

$$
\check{R}_{12}(u) \check{R}_{23}\left(\frac{u+v}{1+\beta^{2} u v}\right) \check{R}_{12}(v)=\check{R}_{23}(v) \check{R}_{12}\left(\frac{u+v}{1+\beta^{2} u v}\right) \check{R}_{23}(u) .
$$

In this paper, we focus on 3-dimensional space. The reduced relativistic YBE reads

$$
A(u) B\left(\frac{u+v}{1+\beta^{2} u v}\right) A(v)=B(v) A\left(\frac{u+v}{1+\beta^{2} u v}\right) B(u) .
$$

Let the unitary Yang-Baxter matrix take the form

$$
\left\{\begin{array}{l}
A(u)=\rho(u)\left(I+F(u) E_{A}\right) \\
B(u)=\rho(u)\left(I+F(u) E_{B}\right) .
\end{array}\right.
$$

Following Xue et al. [38], we obtain

$$
\left\{\begin{array}{l}
F(u)=\frac{e^{-2 i \theta}-1}{d} \\
e^{-2 i \theta}=\frac{\beta^{2} u^{2}+2 i \varepsilon \beta u \sqrt{d^{2} /\left(4-d^{2}\right)}+1}{\beta^{2} u^{2}-2 i \varepsilon \beta u \sqrt{d^{2} /\left(4-d^{2}\right)}+1}
\end{array}\right.
$$

where the new parameter $\theta$ is real. Let $\rho(u)=e^{i \theta}$. The Yang-Baxter matrix can be rewritten in the following form

$$
\left\{\begin{array}{l}
A\left(\theta, \varphi_{1}, \varphi_{2}\right)=e^{i \theta} I-f(\theta) E_{A} \\
B\left(\theta, \varphi_{1}, \varphi_{2}\right)=e^{i \theta} I-f(\theta) E_{B}
\end{array}\right.
$$

where $f(\theta)=2 i \sin \theta / d$.

The Yang-Baxter matrix depends on three parameters: the first is $\theta$ ( $\theta$ is time-independent); the others are $\varphi_{i},(i=$ $1,2)$ contained in the matrix $E$. In physics the parameter $\varphi_{1}$ and $\varphi_{2}$ are flux which depends on time $t$. Usually take $\varphi_{i}=\omega_{i} t,(i=1,2)$ and $\omega_{i}$ are the frequency. Operators $A\left(\theta, \varphi_{1}, \varphi_{2}\right)$ and $B\left(\theta, \varphi_{1}, \varphi_{2}\right)$, satisfying $B\left(\theta, \varphi_{1}, \varphi_{2}\right)=$ $U A\left(\theta, \varphi_{1}, \varphi_{2}\right) U^{-1}$, are unitary operators $\left(A\left(\theta, \varphi_{1}, \varphi_{2}\right)^{\dagger}=A\left(\theta, \varphi_{1}, \varphi_{2}\right)^{-1}, B\left(\theta, \varphi_{1}, \varphi_{2}\right)^{\dagger}=B\left(\theta, \varphi_{1}, \varphi_{2}\right)^{-1}\right)$.

To simplify the following discussion, we will restrict attention to the case $\varphi_{1}=-\varphi_{2}=\varphi$. Following Ge et al. [13], we can obtain Yang Baxter Hamiltonian through the Schrödinger evolution of the states

$$
\hat{H}=i \hbar \frac{\partial B(\theta, \varphi)}{\partial t} B^{\dagger}(\theta, \varphi)
$$

where $\varphi$ be time dependent as $\varphi=\omega t$ and $\theta$ be time independent.

For convenience, we introduce the Gell-Mann matrices $I_{\lambda}[39]$, a basis for $s u(3)$ algebra. Such matrices satisfy $\left[I_{\lambda}, I_{\mu}\right]=i f_{\lambda \mu \nu} I_{\nu},(\lambda, \mu, \nu=1,2, \ldots, 8)$, where $f_{\lambda \mu \nu}$ are the structure constants of $s u(3)$. We denote $I_{ \pm}=I_{1} \pm i I_{2}$ 
$V_{ \pm}=I_{4} \mp i I_{5}, U_{ \pm}=I_{6} \pm i I_{7}$ and $Y=\frac{2}{\sqrt{3}} I_{8}$. Let

$$
\left\{\begin{aligned}
S_{+}= & \zeta\left(-i\left(d^{2}-d-1\right)^{1 / 2}\left(e^{-i \theta}+2 i \sin \theta d^{-2}\right) I_{+}+i d^{1 / 2}\left(e^{-i \theta}+2 i \sin \theta d^{-2}\right) U_{-}\right), \\
S_{-}= & \zeta\left(i\left(d^{2}-d-1\right)^{1 / 2}\left(e^{i \theta}-2 i \sin \theta d^{-2}\right) I_{-}-i d^{1 / 2}\left(e^{i \theta}-2 i \sin \theta d^{-2}\right) U_{+}\right), \\
S_{3}= & \frac{1}{2}\left[\left(1+d-d^{2}\right)\left(1-d^{2}\right)^{-1}\left(\frac{I}{3}+\frac{Y}{2}+I_{3}\right)-\left(\frac{I}{3}+\frac{Y}{2}-I_{3}\right)-d\left(1-d^{2}\right)^{-1}\left(\frac{I}{3}-Y\right)\right. \\
& \left.+d^{1 / 2}\left(d^{2}-d-1\right)^{1 / 2}\left(1-d^{2}\right)^{-1}\left(V_{-}+V_{+}\right)\right],
\end{aligned}\right.
$$

where $\zeta=\frac{d^{2}}{\sqrt{\left(d^{2}-1\right)\left(d^{4}-4\left(d^{2}-1\right) \sin ^{2} \theta\right)}}$. These operators satisfy the $s u(2)$ algebra relations $\left(\left[S_{+}, S_{-}\right]=2 S_{3},\left[S_{3}, S_{ \pm}\right]=\right.$ $\left.\pm S_{ \pm},\left(S_{ \pm}\right)^{2}=0, S_{ \pm}=S_{1} \pm i S_{2}\right)$

In terms of the operators (18), the Hamiltonian Eq.(17) can be recast as following

$$
\hat{H}=-4 \omega \hbar \sin \theta\left(d^{2}-1\right)^{1 / 2} d^{-2}\left(\sin \alpha \cos \beta S_{1}+\sin \alpha \sin \beta S_{2}+\cos \alpha S_{3}\right) .
$$

Its eigenvalues are $E_{0}=0, E_{ \pm}=\mp \omega \hbar \cos \alpha$, where $\cos \alpha=\frac{2 \sin \theta \sqrt{d^{2}-1}}{d^{2}}, \beta=\varphi$, here $d \geq 1$. By the way, its Casimir operator is $\kappa=\frac{1}{2}\left(S_{+} S_{-}+S_{-} S_{+}\right)+S_{3}^{2}$. It is easy to find the eigenvalues of $\kappa$ are $\frac{1}{2}\left(\frac{1}{2}+1\right)=\frac{3}{4}$ and $0(0+1)=0$, which correspond to spin- $1 / 2$ and spin- 0 . According to the definition of Berry phase, when $\varphi(t)$ evolves adiabatically from 0 to $2 \pi$, the corresponding Berry phase is

$$
\gamma_{\alpha}=i \int_{0}^{T}\left\langle\Psi_{\alpha}\left|\frac{\partial}{\partial t}\right| \Psi_{\alpha}\right\rangle d t
$$

Noting that Hamiltonian returns to its original form after the time $T=2 \pi / \omega$, we easily obtain the corresponding Berry phases of this Yang-baxter system

$$
\left\{\begin{array}{l}
\gamma_{0}=0, \\
\gamma_{ \pm}= \pm \pi(1-\cos \alpha)= \pm \frac{\Omega}{2},
\end{array}\right.
$$

where $\Omega=2 \pi(1-\cos \alpha)$ is the solid angle enclosed by the loop on the Bloch sphere. The system also equals to spin$1 / 2$ system and spin-0 system. Substituting $\cos \alpha=\frac{2 \sin \theta \sqrt{d^{2}-1}}{d^{2}}$ into Eq.(21), we obtain $\gamma_{ \pm}= \pm \pi\left(1-\frac{2 \sin \theta \sqrt{d^{2}-1}}{d^{2}}\right)$. Substituting $\theta$ with $\frac{\pi}{2}-\theta$, we rewrite Berry phase as follows

$$
\gamma_{ \pm}= \pm \pi\left(1-\frac{2 \cos \theta \sqrt{d^{2}-1}}{d^{2}}\right)
$$

It is worth mentioning that in some papers [13], the Berry phase $\gamma_{ \pm}= \pm \pi(1-\cos \theta)$ of Yang-Baxter system only depends on the spectral parameter $\theta$. It is interesting that in our paper, the Berry phases(22) not only depends on the spectral parameter $\theta$, but also depends on the topological parameter $d$. The Berry phase (Eq.(22)) reduce to $\gamma_{ \pm}= \pm \pi(1-\cos \theta)$ if $d=\sqrt{2}$. The FIG. 1, which corresponds to the Berry phase $\gamma_{+}$. The FIG. 1(a) illustrate the 

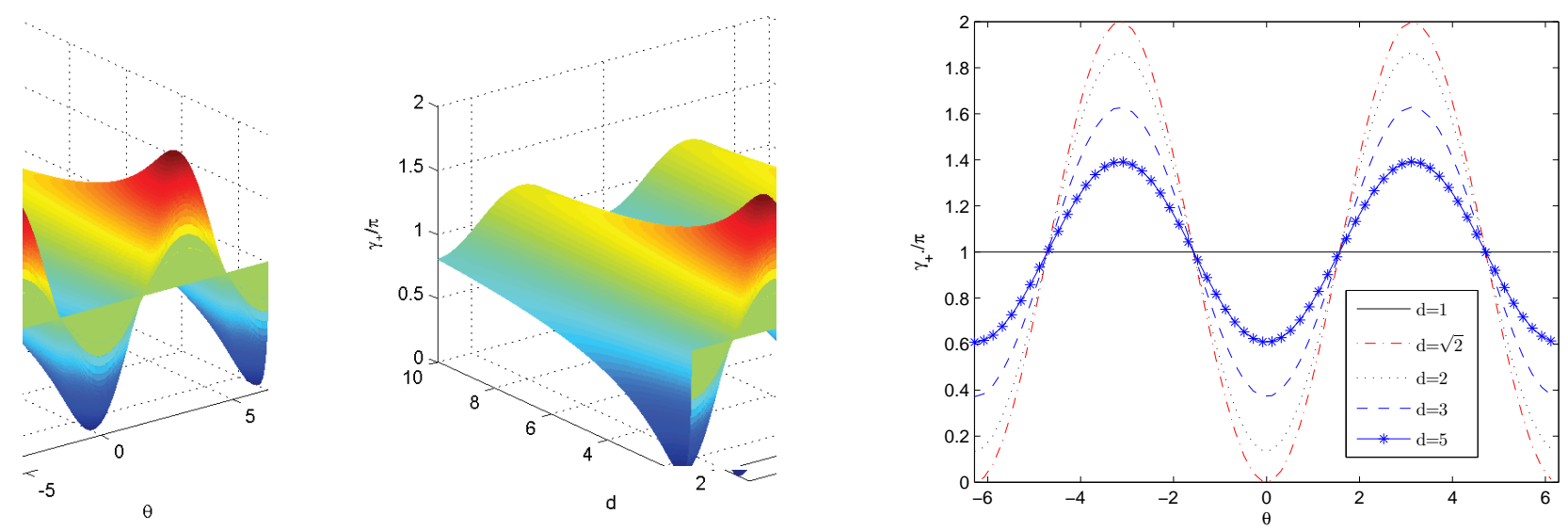

(a)

(b)

FIG. 1. (The left figure shows the Berry phase $\gamma_{+}=\pi\left(1-\frac{2 \cos \theta \sqrt{d^{2}-1}}{d^{2}}\right)$ versus the the topological parameter $d$ and the spectral parameter $\theta$. The right figure, the sectional drawings have also provided with the same values of parameters. $d=1$ (solid line), $d=\sqrt{2}$ (dot-dashed line), $d=2($ dotted line $), d=3$ (dashed line),$d=5$ (star line).

Berry phases Eq.(22) versus the spectral parameter $\theta$ and the topological parameter $d$. The FIG. 1(b) illustrate the Berry phase $\gamma_{+}$versus the spectral parameter $\theta$, when $d$ choice specific values. It is demonstrated that the Berry phase $\gamma_{+}$is maximum (minimum) at parameter $\theta=(2 n+1) \pi(\theta=2 n \pi)$, for a given definit value of $d$. Where $n$ is integer. The maximum (minimum) of $\gamma_{+}$is $\pi\left(1+\frac{2 \sqrt{d^{2}-1}}{d^{2}}\right)\left(\pi\left(1-\frac{2 \sqrt{d^{2}-1}}{d^{2}}\right)\right)$. As $d$ increase, the maximum of $\gamma_{+}$ decreases, the minimum of $\gamma_{+}$increases. The Berry phase $\gamma_{+}$tend to a constant value $\pi$, with $d$ approaches infinity.

\section{SUMMARY}

In this paper we presented BWM-algebra $\left(A, B, E_{A}, E_{B}\right)$ and solution of $\operatorname{YBE}\left(A\left(\theta, \varphi_{1}, \varphi_{2}\right), B\left(\theta, \varphi_{1}, \varphi_{2}\right)\right)$ in 3-dimensional representation which satisify $B=U A U^{-1}, E_{B}=U E_{A} U^{-1}$ and $B\left(\theta, \varphi_{1}, \varphi_{2}\right)=U A\left(\theta, \varphi_{1}, \varphi_{2}\right) U^{-1}$. The evolution of the Yang-Baxter system is explored by constructing a Hamiltonian from the unitary $B(\theta, \varphi)$ matrix. We study the Berry phase of the Yang-Baxter system, and obtain the relationship between topological parameter and Berry phase $\gamma_{ \pm}= \pm \pi\left(1-\frac{2 \cos \theta \sqrt{d^{2}-1}}{d^{2}}\right)$. Then we compare the Berry phase of Yang-Baxter system in Ref.[13] with us, and find the topological parameter d plays a deformation role in the Berry phase. We have been discussing in this paper is still an open problem that will require a deal of further investigations. 


\section{ACKNOWLEDGMENTS}

This work was supported in part by NSF of China (Grant No.10875026)

[1] Yang, C.N.: Some Exact Results for the Many-Body Problem in one Dimension with Repulsive Delta-Function Interaction. Phys. Rev. Lett. 19, 1312 (1967); Yang, C.N.: S matrix for the one-dimensional N body problem with repulsive or attractive delta function interaction. Phys. Rev. 168, 1920 (1968).

[2] Baxter, R.J.: it Exactly Solved Models in Statistical Mechanics. New York: Academic (1982); Baxter, R.J.: Partition function of the Eight-Vertex lattice model. Ann. Phys. 70, 193 (1972).

[3] Temperley, H.N.V., Lieb, E.H.: Relations between the 'Percolation' and 'Colouring' Problem and other Graph-Theoretical Problems Associated with Regular Planar Lattices: Some Exact Results for the 'Percolation' Problem. Proc. Roy. Soc. London, A 322, 251 (1971).

[4] Korepin, V.E., Bogoliubov, N.M., Izergin, A.G.: Quantum Inverse Scattering Method and Correlation Functions. Cambridge University Press (1993)

[5] Kauffman, L.H.: Knots and Physics. Singapore: World Scientific Publ Co Ltd. (1991)

[6] Yang. C.N., Ge. M.L., et al.: Braid Group, Knot Theory and Statistical Mechanics (I and II). SingaporeWorld Scientific Publ Co Ltd. (1989) and (1994).

[7] Baxter, R.J.: The inversion relation method for some two-dimensional exactly solved models in lattice statistics. J.Stat.Phys. 28, 1 (1982); Owczarek, A.L., Baxter, R.J.: A Class of Interaction-Round-a-Face Models and Its Equivalence with an Ice-Type Model. J.Stat.Phys. 49, 1093 (1987); Batchelor, M.T., Barber, M.N.: Spin-s quantum chains and Temperley-Lieb algebras. J. Phys. A 23, L15 (1990).

[8] Batchelor, M.T., Kuniba, A.: Temperley-Lieb lattice models arising from quantum groups. J. Phys. A 24, 2599 (1991).

[9] Li, Y.Q.: Yang Baxterization.J. Math. Phys. 34, 2 (1993).

[10] Kauffman, L.H., Lomonaco Jr, S.J.: Braiding Operators are Universal Quantum Gates. New J. Phys. 6, 413 (2004).

[11] Kitaev, A.Y.:Fault-tolerant quantum computation by anyons. Ann. Phys. 303, 2 (2003).

[12] Franko, J., Rowell, E.C., Wang, Z.: Extraspecial 2-Groups and Images of Braid Group Representations. J. Knot Theor Ramif. 15, 413 (2006).

[13] Chen, J.L., Xue, K., Ge, M.L.: Braiding transformation, entanglement swapping, and Berry phase in entanglement space. Phys. Rev. A. 76, 042324 (2007); Chen, J.L., Xue, K., Ge, M.L.: Berry phase and quantum criticality in Yang-Baxter systems. Annals of Physics 323, 2614 (2008). 
[14] Sun, C.F., et al.: Thermal entanglement in the two-qubit systems constructed from the Yang-Baxter R-matrix. International Journal of Quantum Information 7, 879 (2009).

[15] Wadati, M., Deguchi, T., Akutsu, Y.: Exactly solvable models and knot theory. Phys. Rep. 180, 247 (1989).

[16] Birman .J, Wenzl .H.: Braids, link polynomials and a new algebra. Trans. A.M.S. 313, 249 (1989); Murakami .J.: The Kauffman Polynomial of Links and Representation Theory. Osaka J. Math. 24, 745 (1987).

[17] Abramsky, S.: Temperley-Lieb Algebra: From Knot Theory to Logic and Computation via Quantum Mechanics. e-print quant-ph/0910.2737.

[18] Berry, M.V.: Quantal phase factors accompanying adiabatic changes. Proc. R. Soc. Lond. Ser. A 392, 45 (1984).

[19] Aharonov, Y., Anandan, J.: Phase change during a cyclic quantum evolution. Phys. Rev. Lett. 58, 1593 (1987).

[20] Sjöqvist, E., Pati, A.K., Ekert, A., Anandan, J.S., Ericsson, M., Oi, D.K.L., Vedral, V.: Geometric Phases for Mixed States in Interferometry. Phys. Rev. Lett. 85, 2845 (2000).

[21] Samuel, J., Bhandari, R.: General Setting for Berry's Phase. Phys. Rev. Lett. 60, 2339 (1988).

[22] Tong, D.M., Sjöqvist, E., Kwek, L.C., Oh, C.H.: Kinematic Approach to the Mixed State Geometric Phase in Nonunitary Evolution. Phys. Rev. Lett. 93, 080405 (2004).

[23] Wilczek, F., Zee, A.: Appearance of Gauge Structure in Simple Dynamical Systems. Phys. Rev. Lett. 52, 2111 (1984).

[24] Korepin, V.E., Wu, A.C.T.: Adiabatic Transport Properties and BERRYS Phase in Heisenberg-Ising Ring. International Journal of Modern Physics B 5, 497 (1991).

[25] Appelt, S., Wäckerle, G., Mehring, M.: Deviation from Berrys adiabatic geometric phase in a 131Xe nuclear gyroscope. Phys. Rev. Lett. 72, 3921 (1994).

[26] Jones, J., Vedral, V., Ekert, A., Castagnoli, G.: Geometric quantum computation using nuclear magnetic resonance. Nature 403, 869 (2000).

[27] Duan, L.M., Cirac, J.I., Zoller, P.: Geometric Manipulation of Trapped Ions for Quantum Computation. Science 292, 1695 (2001).

[28] Wootters, W.K.: Entanglement of Formation of an Arbitrary State of Two Qubits. Phys. Rev. Lett. 80, 2245 (1998).

[29] Ekert, A., Ericsson, M., Hayden, P., Inamori, H., Jones, J.A., Oi, D.K.L., Vedral, V.: Geometric quantum computation. J. Mod. Opt. 47, 2501 (2000).

[30] Leibfried, D., et al.: Experimental demonstration of a robust, high-fidelity geometric two ion-qubit phase gate. Nature 422, 412 (2003).

[31] Leek, P.J., et al.: Observation of Berry's Phase in a Solid-State Qubit. science 318, 1889 (2007).

[32] Hu, S.W., Xue, K., Ge, M.L.Optical simulation of the Yang-Baxter equation. Phys. Rev. A 78, 022319 (2008).

[33] Nayak, C., Simon, S.H., Stern, A., Freedman, M., Sarma, S.D.Non-Abelian anyons and topological quantum computation. Rev. Mod. Phys. 80, 1083 (2008). 
[34] Hikami, K.: Skein theory and topological quantum registers: Braiding matrices and topological entanglement entropy of non-Abelian quantum Hall states. Ann. Phys. 323, 1729 (1987).

[35] Cheng, Y.,Ge, M.L., Xue, K.: Yang-Baxterization of braid group representations. Commun Math Phys. 136, 195 (1991).

[36] Ge, M.L., Xue, K.: Trigonometric Yang-Baxterization of colored R-matrix. J. Phys. A: Math. 26, 281 (1993).

[37] Jones, V.F.R.: On a certain value of the Kauffman polynomial. Commun Math Phys. 125, 459 (1987).

[38] Wang, G.C., et al.: Temperley-Lieb algebra, Yang-Baxterization and universal gate. Quantum Information Processing. 9, 699 (2009).

[39] Pfeifer, W.: The Lie Algebras $S U(N)$, An Introduction. Birkhauser Verlag (2003). 